\title{
COLOMBIA: TERRITORIO DE INVERSIÓN EN FUENTES NO CONVENCIONALES DE ENERGÍA RENOVABLE PARA LA GENERACIÓN ELÉCTRICA
}

\section{Colombia: Territory for investment in non-conventional renewable energy to electric generation}

\author{
Wiston Ñustes ${ }^{1}$, Sergio Rivera ${ }^{2}$ \\ ${ }^{1}$ Universidad Nacional de Colombia, Maestría en Ingeniería Eléctrica, Colombia. \\ Email: wanustesc@unal.edu.co \\ ${ }^{2}$ Universidad Nacional de Colombia, Area de Sistemas de Potencia, Smart Grids y Máquinas Eléctricas, \\ Colombia. Email: srriverar@unal.edu.co
}

(Recibido julio 29 de 2016 y aceptado septiembre 14 de 2016)

\begin{abstract}
Resumen
El presente documento corresponde a un Artículo de Reflexión que muestra los diferentes resultados de investigación sobre el panorama del sector eléctrico actual en Colombia y como esto ha llevado a contemplar la inclusión de las Fuentes No Convencionales de Energía Renovable (FNCER) en un corto y mediano plazo. Teniendo como previa discusión la evolución de la industria eléctrica colombiana en las últimas décadas, la problemática actual y la visión de los diferentes agentes del sector eléctrico. Finalmente se presenta las fuentes de financiamiento que se tienen Nacional e Internacionalmente para llevar a cabo proyectos de Generación Distribuida con Fuentes no Convencionales de Energía Renovable en Colombia.
\end{abstract}

Palabras clave: Energía Renovable, Fuentes de Financiación, Generación Eléctrica, Sistema de Potencia Colombiano.

\begin{abstract}
This document corresponds to a reflection paper showing different research results on the picture of the current electricity sector in Colombia and how it has led to contemplate the inclusion of Non-Conventional Renewable Energy (FNCER) in a short and medium term. First, a discussion of the evolution of the electricity industry in Colombia at recent decades is presented, and the current problems and vision of the different agents of the electricity sector are developed. Finally, financing sources (nationally and internationally) needed to carry out projects of Non-Conventional Sources of Distributed Generation and Renewable Energy is presented.
\end{abstract}

Key words: Renewable Energy, Financing sources, Power Generation, Colombian Power System.

\section{INTRODUCCIÓN: EVOLUCIÓN DE LA INDUSTRIA ELÉCTRICA COLOMBIANA}

La evolución de la industria eléctrica en Colombia desde los años 80 hasta el presente fue marcada por los siguientes hitos principales [1-3]:

(i) Evolución de la matriz de generación pasando de un parque energético basado en generación mediante el uso de combustibles fósiles (década de los 80 ) a un parque actual de energía hidroeléctrica (68\%) y energía térmica (28\%), Figura 1 (datos provenientes de [4-8].

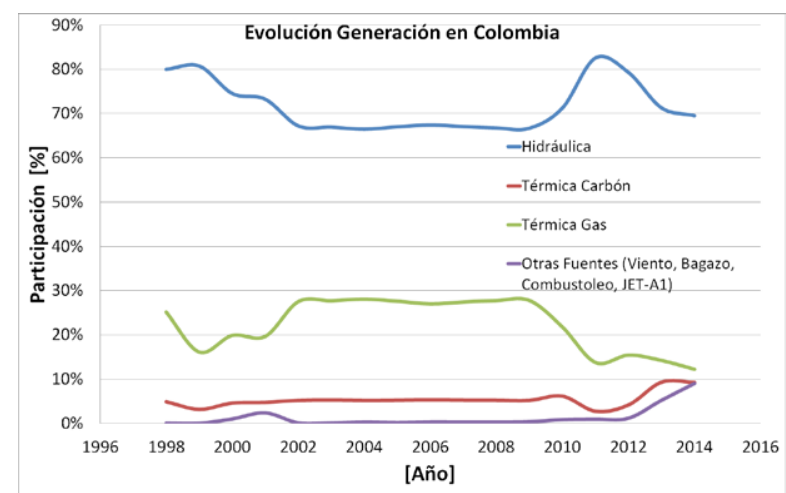

Figura 1. Evolución de la Generación en Colombia.

Cómo citar este artículo:

W. Ñustes, \& S. Rivera, "Colombia: Territorio de inversion en fuentes no convencionales de energía renovable para la generación electrica," Rev. Ingeniería Investigación y Desarrollo, vol. 17 N 1, pp. 37-48, Enero, 2017 
(ii) Evolución de la calidad del servicio técnico y la calidad del producto técnico, asociado al cambio de tecnología [9-11]. Muestra de ello, por ejemplo, es la mejora en la calidad del servicio técnico referente a número de fallas y duración de las fallas, SAIDI y SAIFI, respectivamente, donde se observa las tendencias mostradas en las figuras 2 y 3 (datos provenientes de [9,10,12]. En ellas se presenta para distintos tipos de circuitos de media tensión dados por un grupo determinado (grupo 1 a grupo 4) dependiendo de la cantidad de usuarios conectados [10].

(iii) Evolución de la demanda y de la oferta, donde por ejemplo la tasa anual de crecimiento promedio de la demanda en los últimos 10 años fue de $3.6 \%$, de acuerdo a la Figura 4 (datos provenientes de [4-6].

(iv) Evolución del esquema institucional del sector eléctrico desde lo sucedido a inicios de la década de los 90 cuando la deuda del sector se convirtiera en el principal problema macroeconómico del país [13-14]. Este llegó a representar el $40 \%$ del endeudamiento externo [13].

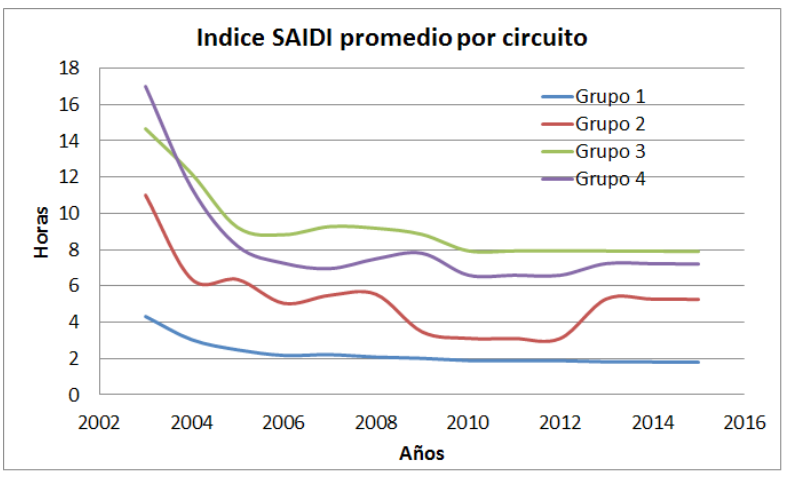

Figura 2. SAIDI promedio por circuito.

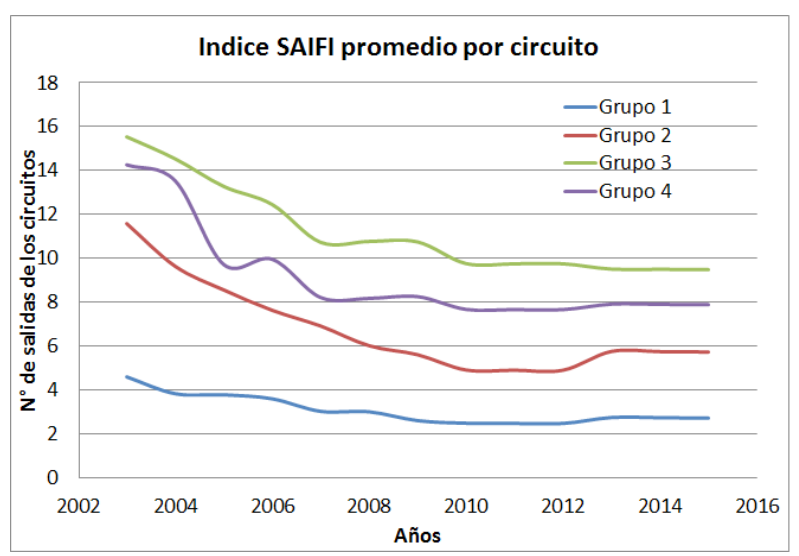

Figura 3. SAIFI promedio por circuito.

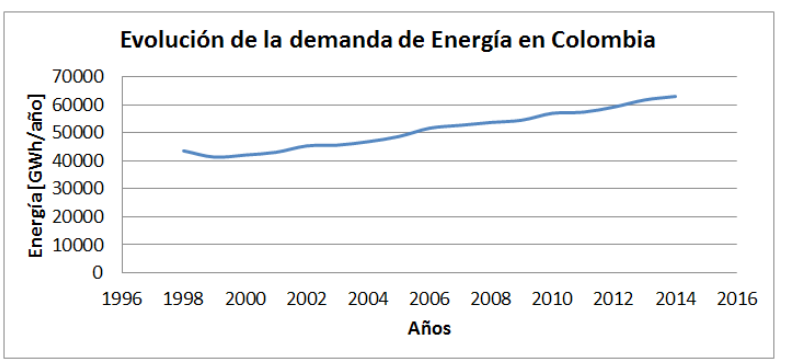

Figura 4. Evolución de la demanda de energía eléctrica en Colombia.

El suceso del racionamiento energético que se extendió entre el 2 de marzo de 1992 y el 1 de abril de 1993 fue el catalizador de un proceso de reforma que reestructuraría por completo el sistema eléctrico. Las leyes 142 y 143 de servicios públicos domiciliarios [15], ley eléctrica de 1994, permitieron definir el papel del Estado, separando las actividades de regulación y control de las acciones propiamente empresariales. 
Adicionalmente, durante la evolución del sector eléctrico colombiano se crearon entidades soporte como $[13,16]$ :

-Unidad de Planeación Minero Energética - UPME, Encargada de establecer requerimientos energéticos de la población y la manera de satisfacerlos; de elaborar y actualizar el Plan Minero Nacional, el plan Energético Nacional y el Plan Indicativo de Expansión del sector Eléctrico.

-Comisión de Regulación de Energía y Gas - CREG, la cual expide las reglas para asegurar la disponibilidad de una oferta energética eficiente, propiciar la competencia, impedir abusos de las entidades prestadoras del servicio y buscar la liberación gradual del mercado eléctrico hacia la libre competencia.

-Superintendencia de Servicios Públicos - SSPD, controla que las empresas que prestan los servicios públicos cumplan con las leyes, las regulaciones y demás actos administrativos que los rigen.

-Centro Nacional de Despacho - CND, es el responsable de realizar las siguientes actividades para la operación integrada de los recursos de del sistema de interconexión nacional (generación, interconexión y transmisión): planeación, supervisión y control.

-Centro Nacional de Operación - CON, encargado de convenir los aspectos técnicos de tal manera que garantiza la operación del sistema de interconexión nacional de una manera segura, confiable y económica.

Finalmente, la prestación y asistencia del servicio de energía eléctrica se definió en cuatro actividades $[13,16]$ : Generación, Transmisión, Distribución y Comercialización. Las cuales se combinan de la siguiente manera: Generación y Comercialización, Transmisión, Distribución y Comercialización, y Comercialización exclusiva [16].

\section{PROBLEMÁTICA ACTUAL}

Colombia posee una capacidad instalada de aproximadamente $16.000 \mathrm{MW}$ de los cuales aproximadamente el $71 \%$ es en parte por recursos renovables principalmente energía generada por centrales hidroeléctricas $[4,5,17]$. La demanda de energía en el 2015 fue de aproximadamente 65.300 GWh con una carga pico de $10.000 \mathrm{MW}$ aproximadamente [17].

Para cubrir esta demanda, la participación de fuentes renovables no convencionales es cercana al 3\% sumado al $4 \%$ proveniente de la generación hidroeléctrica de capacidad menor que $20 \mathrm{MW}$, para un total de aproximadamente un $7 \%$, lo cual es una participación muy pequeña [18]. Por ejemplo, los vientos en Colombia son catalogados como una fuente renovable de alto potencial energético, sin embargo, no se aprovecha ni el $0.4 \%$ de su potencial teórico [4]. La matriz de generación eléctrica actual posiciona al país con una alta tasa de vulnerabilidad del sector eléctrico debido a diferentes factores. Por ejemplo, debido al fenómeno de "El Niño" se ha disminuido recientemente el aporte de la generación hidroeléctrica del país en un $25 \%$. Esta disminución está siendo cubierta por generación térmica que en algunos casos es ineficiente por sus costos variables. Es decir, a principios del 2016 la proporción de generación es del $53 \%$ hidroeléctrica y $43 \%$ térmica (carbón, gas y líquidos). La disminución de generación hidroeléctrica ha implicado que antes de 'El Niño' un MWh costaba aproximadamente USD 90, con el fenómeno pasó a cerca de USD 140 [19]. Adicionalmente, los grandes consumidores son los que enfrentan este aumento de precio debido a que compran en el mercado mayorista, con un sobrecosto para la demanda cercano a los USD 294 millones.

Adicionalmente, el alto costo de los combustibles líquidos y la escasez de gas, han llevado a que algunas generadoras dejen de entregar energía al sistema. Lo cual hace que financieramente sea inviable mantener la producción [19]. Las medidas de mitigación por parte del Gobierno Nacional para estas plantas que están trabajando sin cubrir sus costos son el aumento en la tarifa al usuario, además de las sanciones por el desperdicio energético y las restricciones de exportación de energía a los países vecinos [19].

Por otra parte, en la evolución del sector eléctrico colombiano no se han incorporado ciertas zonas del país. En Colombia aproximadamente el $50 \%$ del territorio nacional corresponde a las Zonas No Interconectadas (ZNI) al Sistema Interconectado Nacional (SIN) [18,20, 21].A pesar de la limitación en recursos económicos, las ZNI del país cuentan con una gran viabilidad de generación de energía eléctrica mediante recursos renovables no convencionales [4,21-22]. 
El contexto descrito revela una gran debilidad en la estructura actual de la matriz eléctrica colombiana y por ende de la confiabilidad del sistema en cuanto al suministro eléctrico, debido a la alta incertidumbre del recurso hidroeléctrico y su vulnerabilidad al "efecto del Niño". Esta realidad es una oportunidad de negocio para diferentes actores que quieran invertir en generación distribuida, redes inteligentes, microredes y parques de generación renovable no tradicionales. De hecho, con mayor penetración de estas tecnologías podrían afrontarse mejor las problemáticas señaladas [17]: complementariedad de distintas fuentes de generación renovables no tradicionales con las tradicionales hidroeléctricas, reducir la vulnerabilidad de los grandes consumidores a las fluctuaciones de los precios y suministro de menores costos y mayor confiabilidad en las ZNI.

\section{VISION DE AGENTES PARTICIPANTES EN EL SIS- TEMA DE POTENCIA COLOMBIANO}

En aras de que el sistema de potencia colombiano sea más robusto ante las problemáticas mencionadas, diferentes agentes actores ((i) Distribuidoras, (ii) Estado (iii) Demanda y (iv) Generadores) están de acuerdo en que deberían darse las inversiones señaladas [17], dado también la reducción en los costos de estas nuevas tecnologías. Para este trabajo de investigación se entrevistaron (mediante reuniones y correos electrónicos) los diferentes agentes del mercado, académicos y profesionales relacionados, como también a ASOCODIS (Asociación Colombiana de Distribuidores de Energía Eléctrica). Este gremio de empresas distribuidoras de energía agrupa las 23 principales empresas del país, que atienden el $98 \%$ de los usuarios del país y con presencia en $96 \%$ de los municipios de Colombia. De esta manera se logra percibir la siguiente visión para cada uno de ellos, complementando la visión presentada de Colombia en el trabajo presentado en [17]:

\subsection{Visión de las Distribuidoras de Energía Eléctrica}

Las oportunidades de negocio que perciben los agentes del mercado en el corto, mediano y largo plazo respecto de la distribución de energía en Colombia pueden ser consideradas desde los siguientes enfoques (entrevista a ASOCODIS):
-El Estado desde hace algunos años inició la privatización de compañías en las cuales era accionista mayoritario, existiendo aun algunas de ellas en poder del estado. Como oportunidad de negocio se visualiza el tema de ventas de compañías distribuidoras de energía a inversionistas privados. Esto representa una oportunidad de negocio no solo para inversionistas nacionales sino también para internacionales. La condición para los inversores es que su participación en el mercado no supere el $25 \%$ de la demanda nacional para evitar conductas monopólicas.

-La distribución de energía se puede considerar como una actividad monopólica con una regulacion y con una rentabilidad establecida. Sus oportunidades de negocio están limitadas a la expansión de sus sistemas eléctricos con el objetivo de atender un mayor crecimiento de la demanda (vertical de los usuarios existentes y horizontalmente de nuevos usuarios) y a eventuales medidas de eficiencia cuando mejoran ciertos indicadores. Adicionalmente, con las nuevas regulaciones implementadas, se habilitó la posibilidad de que empresas distribuidoras puedan desarrollar proyectos del Sistema de Transmisión Regional en mercados de otras distribuidoras del país, lo cual implica una nueva posibilidad de negocio para estas empresas ampliando sus oportunidades.

-En la actualidad surge un gran reto para las Empresas Distribuidoras luego de la entrada en vigencia de la ley 1715 de 2014 (ley que regula la integración de energías renovables no convencionales al sistema energético nacional), pues en la medida que se introduzcan masivamente este tipo de nuevos actores a los Sistemas Eléctricos de los Distribuidores, es posible que la demanda atendida disminuya y estas empresas deban reorientar su negocio involucrándose como una actor principal en el ofrecimiento al usuario de nuevas tecnologías de generación para el suministro del servicio compitiendo con otras tecnologías. De esta forma es posible explotar mucho más el beneficio social que ofrecen las mismas.

-Una gran oportunidad de negocio puede surgir para las Operadoras de Red en la medida que se tenga una mayor penetración de vehículos eléctricos o se utilice este energético para el transporte masivo en varias ciudades principales del país.

De este modo, las distribuidoras pueden ayudar a la crisis energética debido al fenómeno del Niño y los 
problemas ocasionados por el cargo de confiabilidad. Divulgando a sus usuarios los planes de ahorro en los sistemas energéticos y el uso adecuado y de manera eficiente de la energía, con miras a minimizar las posibilidades de racionamiento por crecimiento de la demanda. Existe en este momento regulación para que los usuarios puedan brindar el servicio de "Respuesta a la Demanda", la cual no ha entrado en vigencia. Un adecuado otorgamiento de incentivos podría contribuir también a minimizar riesgos de racionamiento.

La problemática evidenciada en los últimos meses de 2015 y comienzo del 2016, sobre el manejo que se le ha dado al por Cargo por Confiabilidad, es un tema sensible para el sector y afecta la formación del precio de generación a los usuarios que atienden las distribuidoras. Las Operadoras de Red han tomado posición frente al tema más como comercializadores de energía que propiamente como distribuidores de energía, pues las condiciones de sobreprecio y cobertura afectan al usuario final, quien permite a través del pago del servicio, que se remuneren todas las actividades de la cadena.

Por otra parte, las distribuidoras piensan que los incentivos que se están otorgando en la Ley 1715 de 2014 pueden ser los adecuados, sin embargo, se requiere un tiempo prudencial para que estén en vigencia y se implementen todas las normativas que deben emitirse de acuerdo a lo contemplado en la ley.

\subsection{Visión Estratégica del Estado, Entes Reguladores y de Planeación}

El estado consciente de la potencialidad de: la fuentes no convencionales de energía, las redes inteligentes y microredes para hacer frente a las problemáticas señaladas, ha establecido el plan energético nacional a través de la UPME para los años 2015 a [22], donde se plantean varias estrategias y metas con el fin de utilizar recursos de generación no convencionales, con especial énfasis e interés hacia las comunidades rurales y áreas aisladas del sistema interconectado nacional [21]. Dicho interés incentiva la implementación de microredes con el fin de satisfacer las necesidades particulares que se tengan en las diferentes ZNI.

Con el fin de garantizar lo estipulado en el plan energético nacional se han creado varias herramientas legales que lo permitan, como lo es la ley 1715 de 2014 de la CREG en la cual se estipulan los siguientes puntos importantes [23]:

- Derecho a reducir anualmente de su renta $50 \%$ del valor total de la inversión realizada durante los cinco (5) años siguientes a la realización de dicha inversión.

- Exención del IVA para la importación de recursos y/o equipos destinados a proyectos basados en fuentes de energía no convencionales.

- Exención de aranceles en la importación de materiales e insumos y equipos a utilizar en proyectos con fuentes de energía no convencionales.

- Equipos, maquinaría y obras civiles destinadas a proyectos de generación basados en fuentes de energía no convencionales gozarán de depreciación acelerada.

Los beneficios tributarios y arancelarios expuestos en esta ley son otorgados y respaldados mediante el estatuto tributario, así como también se extienden los beneficios a instituciones de educación superior para investigación y se amplían los beneficios otorgados a proyectos basados en fuentes de energía no convencionales.

El Estado destaca que las leyes relacionadas con energía renovable brindan las bases para los primeros proyectos, $\mathrm{y}$ por ende investigaciones en redes inteligentes $\mathrm{y}$ microredes [4,17]. Con los planes que menciona el estado se aspiran a escenarios donde por ejemplo en el 2020 el 8\% de la demanda sea cubierta con generación renovable no convencional, y que en el 2030 sea del $15 \%[17,18]$.

\subsection{Necesidad de Participación Activa de la Demanda}

Según el gremio que reúne a los grandes consumidores de energía (Asoenergia), el país necesita incorporar del orden de $2.000 \mathrm{MW}$ de potencia firme en generación de bajo costo. Esta apreciación proviene del aumento de precios que los consumidores sufrieron debido al fenómeno de "El Niño". Una solución para este déficit de capacidad instalada de bajo costo es la inversión en generación renovable no convencional, microredes y la demanda puede participar invirtiendo y participando 
como autogeneradores. Para incentivar la figura de autogeneradores existen regulaciones en Colombia donde se establecen los lineamientos de política energética en materia de entrega de excedentes de autogeneración y donde se establecen los límites máximos de potencia de autogeneración a pequeña escala [24].

Por otra parte, en Colombia existen regulaciones que buscan incentivar la respuesta de la demanda fundamentado en un esquema de desconexión voluntaria de demanda. A pesar de que existen estas regulaciones la demanda no las conoce ni utiliza. El uso de esta herramienta conllevaría a que la demanda podría reducir su consumo en respuesta a la señal de precio del mercado mayorista de energía. Los incentivos para la demanda estarán estipulados en contratos bilaterales con los comercializadores, experimentando ahorros en las cuentas de energía eléctrica, principalmente por reducción de consumos en las horas pico.

\subsection{Visión de las generadoras}

En aras de promover las Fuentes no Convencionales de Energía Renovable (FNCER) en el país, los agentes generadores se encuentran en una posición que favorece el plan energético de expansión para los años 2015 a 2029 realizado por la UPME [25]. El cual estipula estrategias y metas que permiten el aprovechamiento de los recursos no convencionales a lo largo del territorio colombiano, con especial énfasis e interés hacia las comunidades rurales y áreas aisladas del sistema interconectado nacional [21].

Se debe considerar que para que estas fuentes renovables sean parte fundamental de un mercado eléctrico mayorista, los Entes Reguladores y de Planeación deben fortalecer un marco regulatorio que lo permita. Y así, crear condiciones para que las empresas internacionales que quieren ingresar al país para producir energías renovables se decidan a hacerlo [26]. Lo que contribuiría a una mejora en la confiabilidad del servicio de generación teniendo recursos más eficientes como son estas fuentes limpias [21].

\section{FUENTES DE FINANCIACIÓN DE LA GENERACIÓN NO CONVENCIONAL DE ENERGÍA RENOVABLE}

Como se puede apreciar en las secciones anteriores, la necesidad en Colombia de profundizar en las nuevas fuentes de energía no convencionales (FNCER), es un hecho. La alta dependencia de los recursos hídricos pone en riesgo el sustento eléctrico del país periódicamente; lo cual, es evidente que con el paso del tiempo el impacto de las sequias en el territorio colombiano debido al calentamiento global es cada vez mayor. Adicionalmente el potencial energético es altamente aprovechable siempre y cuando se proporcionen los diferentes instrumentos por parte de los entes gubernamentales, que permitan el desarrollo de las diferentes tecnologías como Eólica, Solar, Geotérmica, Biomasa, entre otras. Un gran paso en este proceso de la inclusión de las FNCER es la ley 1715 de 2014. La cual, define como su objeto [23]: "Promover el desarrollo y la utilización de las fuentes no convencionales de energía, principalmente aquellas de carácter renovable, en el Sistema energético nacional, mediante su integración al mercado eléctrico, su participación en las Zonas no interconectadas y en otros usos energéticos como medio necesario para el desarrollo económico sostenible, la reducción de emisiones de gases de efecto invernadero y la seguridad del abastecimiento energético...". De este objetivo se tienen los instrumentos y elementos para cumplir con este propósito, los cuales han sido clasificados en económicos, normativos y otros [23].

Del mismo modo, el relativo subdesarrollo de las tecnologías no convencionales de energía en el país, radica en los diferentes factores limitantes: políticos, económicos y sociales. Hasta ahora, debido a los costos de inversión asociados, abundancia energética de los recursos convencionales y dificultades socioculturales y políticas, los encargados de tomar las decisiones del sector energético no han logrado trazar una hoja de ruta que fortalezca la inclusión de las FNCER. En la mayoría de los casos, las áreas más viables para el aprovechamiento de las FNCER se encuentran localizadas en sitios alejados de redes eléctricas, comunicaciones y vías de acceso, lo cual dificulta la construcción de estos proyectos [21-22]. 
Ante estas limitantes, los mecanismos que permiten mitigar las barreras para incursionar las fuentes renovables, son los esquemas de financiamiento a proyectos relacionados con: generación mediante energías limpias, cambio climático y medio ambiente. Estos recursos pueden ser proporcionados por entidades internacionales o locales dependiendo del enfoque que se tenga. A continuación, se describen las principales fuentes de financiamiento:

\subsection{Fuentes Internacionales de Financiamiento}

Diferentes entidades internacionales de la banca multilateral y del sistema de las Naciones Unidas cuentan con programas para el desarrollo de proyectos de Fuentes de Energía Renovable. Por ejemplo, el banco alemán Kreditanstalt für Wiederaufbau (KfW) se caracteriza por el financiamiento de proyectos innovadores en Energías Renovables en Alemania y otros países del mundo. Las dos filiales IPEX (Banco para el Financiamiento Internacional de Proyectos y Exportaciones) y DEG (Corporación Alemana para la Inversión y el Desarrollo), promueven la financiación en Colombia. La primera encaminada al apoyo de compra de productos y tecnologías alemanas en todos los sectores económicos, y la segunda al financiamiento de proyectos de gran envergadura entre 4 y 10 años de duración y créditos hasta 40 millones de Euros. A través del KfW es posible hacer la conexión con otros programas de financiación de bancos como: el Banco Europeo de Inversiones o el Banco Europeo para la Reconstrucción y el Desarrollo. La Sociedad Alemana de Cooperación Internacional, financia hasta el $50 \%$ de proyectos que fortalezcan los temas de Medio Ambiente y Clima en América del Sur. El aporte de esta organización también está enfocado en el acompañamiento de proyectos que se encuentran en desarrollo y desean tener apoyo de personal experto calificado [27].

Por otro lado, el Gobierno Americano por medio de la Agencia de los Estados para el Desarrollo Internacional (USAID), también apoya pequeños proyectos con fuentes renovables en Colombia. El Banco Interamericano de Desarrollo (BID) ofrece financiamiento a largo plazo para obras que superan los 10 millones de USD por medio de Structured and Corporate Finance Department (SCF). La IIC (Inter-American Investment Corporation /IBD GROUP), de la cual Colombia es miembro, ofrece a las PYMES medios de recibir capital propio y financiamiento de crédito de 1 a 15 millones USD [27]. El Programa
FINPYME Credit, se enfoca en proyectos menores que están entre 100.000 y 600.000 USD [27]. La Fundación Interamericana (IAF) cuenta con recursos donativos por parte del gobierno de los Estados Unidos para promover ideas creativas que ayuden a mejorar la calidad de vida de poblaciones en Zonas No Interconectadas en Latinoamérica y el Caribe.

La Corporación Andina de Fomento (CAF), promueve el desarrollo de sistemas energéticos a través de financiamiento en proyectos dentro de las siguientes líneas estratégicas: energías renovables, electrificación rural e innovación tecnológica $[13,28]$. Finalizado el año 2013, Colombia solicitó a la CAF 140 millones USD para el desarrollo de generación con energía Solar, Eólica, Hidroeléctrica, Termoeléctrica y en el fortalecimiento de la infraestructura del sistema de Transmisión. En el momento la CAF ofrecen líneas de crédito por hasta 200 millones de dólares y 18 años de plazo. Lo cual es una buena alternativa para seguir promoviendo proyectos con energías renovables en el país.

El FOMIN (Fondo Multilateral de Inversiones del BID) es un fondo para la financiación de propuestas enfocadas en la incorporación de FNCER en áreas rurales de tal manera que se generen esquemas productivos y se ayuden a mitigar el uso de fuentes contaminantes [27]. Este fondo aporta recursos a través de donativos no reembolsables y orientados en su mayoría a la pequeña y mediana empresa [27].

El Banco Mundial por su parte a través del Programa de Asistencia para la Gestión del Sector de Energía (ESMAP) ayuda a diferentes países a estructurar un sistema energético con recursos renovables, desarrollando políticas ambientales y facilitando el apoyo financiero para cumplir el objetivo. BioCarbon Fund que también hace parte del Banco Mundial, financia proyectos que ayuden a conservar el carbono en los sistemas agrícolas y forestales.

EL Programa de Naciones Unidas para el Desarrollo (PNUD), financia esquemas que fortalezcan y ayuden a preservar el medio ambiente. Estos recursos son gestionados por el Fondo Mundial y está dirigido a todos los grupos organizados de la sociedad civil, comunidades gubernamentales o académicas que tengan proyectos encaminados con este fin. 
GEERED (Global Energy Efficiency and Renewable Energy Fund), es una asociación que gestiona fondos del sector público para la inversión de proyectos en energías limpias en países emergentes. La Agencia de Cooperación Internacional del Japón (JICA), brinda apoyos no reembolsables para el crecimiento social y económico de las regiones en vía de desarrollo. Colombia ha sido favorecida en formación y capacitación principalmente en energías geotérmicas.

El grupo ProCredit financia proyectos amigables con el ambiente (proyectos verdes), con el objetivo de aumentar las inversiones en fuentes renovables, eficiencia energética y gestión sostenible. EI financiamiento verde es parte de una estrategia integral que ProCredit está adoptando con el objetivo de mejorar su impacto ambiental a nivel interno y externo. Con lo cual se pretende contribuir a mitigar el cambio climático [29].

\subsection{Fuentes Nacionales de Financiamiento}

\section{Fondos Estatales}

Como parte del propósito para el desarrollo de las Fuentes no Convencionales de Energía Renovable en el país, existen entidades nacionales que tienen esquemas de financiamiento y apoyan los proyectos de energías renovables. Los fondos estatales a los que se pueden tener acceso son: FAZNI (Fondo de Apoyo Financiero para la Energización de Zonas No Interconectadas), SGR (Sistema General de Regalías), FAER (Fondo de Apoyo Financiero para la Energización de las Zonas Rurales Interconectadas), FECF (Fondo Especial Cuota de Fomento) y FENOGE (Fondo de Energías Renovables y Gestión Eficiente de la Energía) [24].

Los proyectos que deseen tener el apoyo de estos fondos, deben exponer la solución a un problema de servicio energético concertado con la comunidad objetivo y estudios técnicos, ambientales y económicos que muestren la viabilidad del mismo. Se evalúa por las entidades encargadas de los fondos y al ser aprobada la solicitud, se obtiene hasta el $100 \%$ de financiamiento del proyecto, el cual pasa a ser no una forma de crédito sino una inversión del Estado.

Los Fondos FANZI y FAER financian programas que desarrollen nueva infraestructura eléctrica de tal manera que la cobertura del servicio de energía se amplíe y se mejore la demanda en las Zonas no Interconectadas del país y zonas rurales interconectadas. Con el SGR se tienen los siguientes campos: construcción, ampliación, optimización, rehabilitación, montaje, instalación y puesta en servicio de infraestructura en generación eléctrica para las ZNI, generación en el SIN, Alumbrado Público y líneas de transmisión y distribución [27].

FENOGE por su parte fue creado por el artículo 10 de la ley 1715 de 2014 con el fin de promocionar, estimulare incentivar el desarrollo de las actividades de producción y utilización de Fuentes no Convencionales de Energía. Para asegurar la diversificación del abastecimiento energético pleno y oportuno [23,24]. De acuerdo a esto, FENOGE financia recursos a programas que van desde la implementación de autogeneradores en pequeña escala basados en FNCER, hasta proyectos de implementación de nuevas tecnologías de eficiencia energética [27].

\section{Otras Fuentes de Financiación en Colombia}

FINDETER (Financiera de Desarrollo Territorial S.A.) es el banco de desarrollo para la infraestructura sostenible del país, el cual presta el servicio de créditos de redescuentos para financiar proyectos de infraestructura pública y privada en Colombia. Recibe ayuda y asistencia de Latin American Investment Facility y de la Unión Europea. Uno de sus objetivos es apoyar la producción, transmisión, transporte, ahorro, distribución y comercialización de energías renovables en el país [24]. Para el año 2015 se contó con una línea específica que financió proyectos de penetración de energías renovables y expansión eficiente de alumbrado público e Iluminación Interior o Exterior [22]. La línea contó con cien mil millones de pesos COP con plazos de hasta 8 años y periodo de gracia a capital hasta 2 años.

Bancoldex-Colciencias es un fondo que apoya la investigación, el desarrollo tecnológico y la innovación de las tecnologías de Fuentes no Convencionales de Energía Renovable en Colombia. El incentivo que se destaca es el prepago de las primeras cuotas de capital correspondientes entre el $25 \%$ y $50 \%$ del total del crédito para proyectos de innovación. La financiación puede alcanzar el $80 \%$ del monto total, de tal manera que la empresa emprendedora contribuya con el $20 \%$ restante. Los temas de interés son el diseño, desarrollo de procesos y servicios que fortalezcan la eficiencia energética. Otro tema de interés es la 
optimización, simulación y control de procesos. Dentro de los más destacados intereses está el desarrollo de nuevas tecnologías en fuentes no convencionales de energía [30]. Por otro lado, el programa aProgresar de Bancoldex, cuenta con dos líneas de crédito especiales que trabaja bajo el mecanismo de redescuento. El primero es aProgresar-Capitalización que fortalece las MIPYMES de todos los sectores económicos en sus planes de mejoramiento. El cupo para este programa es de aproximadamente 110 mil USD. El Segundo es aProgresar - Modernización, Innovación, el cual cuenta con recursos para proyectos de innovación y desarrollo tecnológico que superan los 330 mil USD [30].

Finalmente, Colciencias cuenta en este momento con convocatorias de proyectos de Ciencia, Tecnología e Innovación (CTel). Los cuales están dirigidos a los centros y grupos de investigación que trabajen con el enfoque de las líneas temáticas (Colciencias, 2016). El presupuesto es de $\$ 34$ mil millones de pesos para los tres temas centrales: Construcción de Paz, Crecimiento Verde y Recursos renovables y no renovables. Este tercer eje permite el desarrollo de sistemas de generación localizada y distribuida de energía junto con proyectos de aprovechamiento energético de fuentes limpias [31].

\section{CONCLUSIÓN: UNA OPORTUNIDAD DE INVERSIÓN EN FUENTES RENOVABLES}

Bajo una perspectiva empresarial o de negocio, la inversión en proyectos de generación de energía a partir de fuentes no convencionales es una buena oportunidad en Colombia. Además de contar con las diferentes fuentes de financiación mencionadas, los incentivos tributarios ofrecidos son bastante favorables para incursionar en un mercado que no está siendo explotado.
Por ejemplo, en la comercialización de energía en áreas rurales o fuera del sistema interconectado, no se presenta actualmente competencia en lo absoluto. La implementación de microrredes resulta una alternativa muy conveniente para poder lidiar con las problemáticas señaladas anteriormente.

Ello se fundamenta en que partiendo de un diseño general se pueden adaptar a las diferentes $\mathrm{ZNI}$, de acuerdo a los recursos energéticos disponibles en cada caso. Es posible proveer de energía eléctrica a dichas comunidades, a partir los mismos recursos renovable que se disponen. Por ejemplo, el potencial energético eólico de Colombia es 29.500 MW, el solar es de 27,7 kWh/m2/día, y el geotérmico 2000 MW. De los cuales, solo se tiene instalado aproximadamente $630 \mathrm{MW}$.

De acuerdo a lo señalado en esta sección, la conclusión es que los diferentes actores del sector eléctrico colombiano deben preparar planes de inversión donde se contemple alta penetración de generación distribuida, redes inteligentes, microredes y parques de generación renovable no convencionales (Figura 5). De esta forma se puede lograr un sistema más robusto ante las problemáticas descritas en la segunda sección: combinación de generación, poca presencia de fuentes alterativas de generación renovable, vulnerabilidad de los grandes consumidores a las fluctuaciones de los precios y confiabilidad en el suministro a las ZNI.

Todo esto muestra el buen panorama que se tiene para la inversión de Fuentes no Convencionales de Energía Renovable en Colombia. La motivación es amplia por parte de las diferentes organizaciones que buscan el crecimiento energético limpio en nuestro país en un corto mediano y largo plazo. Con esto, se invita a hacer parte de este desarrollo nacional. 


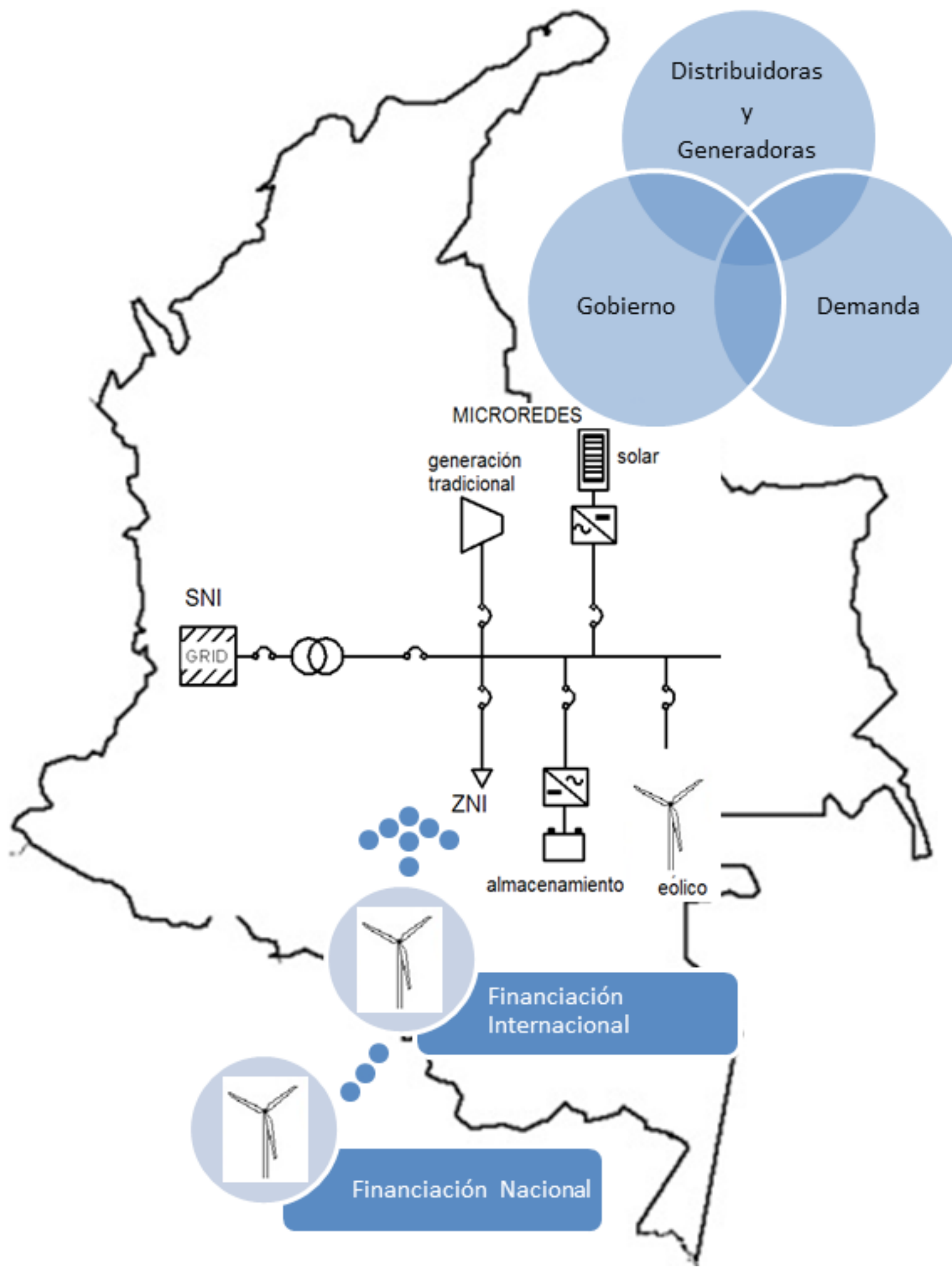

Figura 5. Oportunidad de Inversión en Fuentes Renovables en Colombia. 


\section{REFERENCIAS}

[1] S. Bello, \& R. Beltran, “Caracterización y pronóstico del precio spot de la energía eléctrica en Colombia," Rev. maest. derecho econ. vol. 6, pp. 293-316, 2010.

[2] R.D. Cruz, H. Torres, M.M. Montoya, J. Barrientos, L.M. Pineda, L.M. Niebles, et al., "Caracterización del sector eléctrico colombiano," Servicio Nacional de Aprendizaje, Medellin: SENA, jun. 2013. Diponible en: file://C:/Users/UPTC/Downloads/3058. pdf

[3] S. Carvajal \& D. Marín," Impacto de la generación distribuida en el sistema eléctrico de potencia colombiano: un enfoque dinámico," Tecnura, vol. 17, $\mathrm{n}^{\circ} 35$, pp. 77-89, 2013. DOI: https://doi. org/10.14483/udistrital.jour.tecnura.2013.1.a07.

[4] E.E. Gaona, C.L. Trujillo, \& J. A. Guacaneme, "Rural microgrids and its potential application in Colombia," Renew. Sustain. Energy Rev., vol. 51, pp. 125-137, 2015. DOI: https://doi.org/10.1016/j. rser.2015.04.176.

[5] S. Ortiz, "Generación Eléctrica," Mundo Eléctrico, vol. 99, pp. 1-98, 2015.

[6] J. Pinto Nolla, "Mejora de Acceso a Mercados Energéticos Fase I - Colombia, Estudio Integral de la Situación Actual y Perspectivas del Mercado Energético de Colombia," Organización Latinoamercana de Energía, Quito: OLADE, Oct. 2013. Disponible en: http://www.olade.org/sites/default/ files/CIDA/Colombia\%20Informe\%20Final\%200ctubre\%20V3.pdf

[7] UPME, Boletín Estadístico de Minas y Energia 1990 - 2010, Bogota: UPME, 2010.

[8] UPME, Boletín Estadístico de Minas y Energía 2010 - 2015, Bogota: UPME, 2015

[9] O. Agudelo, \& L. Arango, "Evolución de la confiabilidad y la eficiencia en el sector eléctrico Colombiano." Ventana Informática, vol. 24, pp. 115-133, 2011.

[10] A. Rueda, I. Bonilla, \& Y. Garzón, “Modelo de confiabilidad basado en la norma de gestión de riesgos iso 31000 para empresas de distribución de energía eléctrica en circuitos radiales," Tecnura, vol. 19, pp. 41-50, 2015. DOI: http://dx.doi.org/10.14483/ udistrital.jour.tecnura.2015.ICE.a04

[11] I. Durán, L. Rosero, A. Pavas, \& O. Duarte, “Comparison of maintenance scheme effects on power transformer reliability performance," Ingeniería e Investigación, vol. 35, pp. 73-81, 2015. DOI:ht-
tp://dx.doi.org/10.15446/ing.investig.v35n1Su p.53435

[12] UPME, "Informe sectorial sobre la evolución de la distribución y comercialización de energía eléctrica en colombia: balance de sucesos y estadísticas 1998-2010,"Medellin: UPME, 2011

[13] A. Briceño, "Colombia: análisis del sector eléctrico,"Caracas: Corporación Andina de Fomento CAF, 2006.

[14] L. Vélez, El sector eléctrico colombiano: descripción, situación actual, retos y perspectivas, Agosto, 2015 Retrieved Julio 4, 2016, from: http://luisguillermovelezalvarez.blogspot.com.co/2015/08/ el-sector-electrico-colombiano.html

[15] Régimen Básico, Ley 142 de 1994, Ley 143 de 1994, Ley 689 de 2001, Ley 632 de 2000, Decreto 990 de 2002, Bogotá: Superintendencia de Servicios Públicos Domiciliarios, 2014.

[16] Interconexión Eléctrica S.A, "El sector Eléctrico Colombiano Origenes, Evolución y Retos. Un siglo de desarrollo 1882-1999", Bogotá: Panamericana Formas e Impresos S.A., 2012.

[17] A. Vargas, O. Saavedra, M. Samper, S. Rivera, S., \& R. Rodriguez, "Latin American Energy Markets: Investment Opportunities in Nonconventional Renewables," IEEE Power and Energy Magazine, vol. 14, pp. 38-47, 2016. DOI: https://doi.org/10.1109/ MPE.2016.2573862.

[18] H. García, A. Corredor, L. Calderón, \& M. Gómez, "Análisis costo beneficio de energías renovables no convencionales en Colombia", FEDESARROLLO, Centro de Investigación Económica y Social, Bogotá, 2013.

[19] ACOLGEN. Asociación Colombiana de Generadores de Energía Eléctrica [Online]. Colombia: Bogotá, Jul. 2016 Disponible en: http://www.acolgen.org. $\mathrm{co} /$

[20] J. Moreno, C. Rodríguez, \& R. Suesca, “Generación híbrida de energía eléctrica como alternativa para zonas no interconectadas," Ingeniería, vol. 12, pp. 57-63, 2006.

[21] UPME, Integración de las energías renovables no convencionales en Colombia, Medellin: UPME, 2015.

[22] UPME, Plan de Expansión de Referencia Generación Transmisión 2015 - 2029, Marzo, 2016, Retrieved Julio 5, 2016, from: http://www1.upme. gov.co/sala-de-prensa/fotonoticias/plan-de-expansion-2015-2029 
[23] Congreso de la República de Colombia, Ley 1715 de 2014, Junio, 2016. Retrieved Julio 5, 2016, from: http://www.secretariasenado.gov.co/senado/

[24] Ministerio de Minas y Energía, "Resolución 40393 de 2015_Diario oficial $\mathrm{N}^{\circ} 49.469$ de 30 de marzo de 2015" Ministerio de Minas y Energía, Bogotá, D.C., Marzo, 2015. Retrieved Julio 5, 2016, from: http://www.icbf.gov.co/cargues/avance/docs/resolucion_minminas_40393_2015.htm

[25] UPME, Sistema de Gestión de Información y Conocimiento en Fuentes No Convencionales de Energía Renovable (SGIC - FNCER), (UPME), 2016. Retrieved Julio 5, 2016, from: http://www1.upme. gov.co/sgic/

[26] Centrales Electricas de Nariño S.A. E.S.P. CEDENAR, Sector eléctrico, de acuerdo con nuevo plan de expansión [Online], CEDENAR, Feb. 2016. Retrieved from: http://www.cedenar.com.co/index.php/ productos-servicios/noticias/147-sector-electrico-de-acuerdo-con-nuevo-plan-de-expansion

[27] H. Calderón, "Financiación de Proyectos en el sector de energías renovables en Colombia", Foro de Energías Renovables para zonas no interconectadas, Bogotá, 2014. DOI: http://dx.doi. org/10.14483/udistrital.jour.tecnura.2013.1.a07
[28] CAF - Banco de desarrollo de América Latina, Sistemas energéticos sostenibles [online]. Caracas, 2013 Disponible en: http://scioteca.caf.com/bitstream/handle/123456789/477/Energia\%202015. pdf? sequence $=4 \&$ is Allowed $=y$

[29] Internationale Projekt Consult - IPC GmbH, Un enfoque metodológico destinado a recopilar una lista de medidas estándar de inversión en eficiencia energética que reúnan los requisitos necesarios, Bogotá: ProCredit, 2013.

[30] Bancoldex. A Progresar [Online], julio, 2016. Retrieved Junio 30 de 2016, from: https://www.bancoldex.com/Memorias/Memorias_aProgresar_Financiero.aspx

[31] Colciencias, Convocatoria para proyectos de Ciencia, Tecnología e Innovación y su contribución a los retos de país -2016 [Online], Bogota: Colciencias, 2016.

[32] Comisión de Regulación de Energía y Gas, Resolución No 097 de 2008, 2008.

[33] Ministerio de Minas y Energia, Proyecto de Decreto "Por el cual se reglamenta el Fondo de Energías Renovables y Gestión Eficiente de la Energía - FENOGE", Bogotá: Ministerio de Minas y Energía, 2005. 